\title{
IDEAS DE LOS ALUMNOS ACERCA DEL MOL. ESTUDIO CURRICULAR
}

\section{GRUPO ALKALI:}

\author{
GARCía MARTÍN, J. P.(1), PIZARRo GALÁN, A. M${ }^{\text {a(2) }}$, PERERA CENDAL, F ${ }^{(3)}$, MARTíN DÍAZ, M*
} J.(4), BACAS LEAL, $P$, $^{(5)}$

(1) I. B. "Zurbarán", Badajoz. (2) I. B. "Sta. Eulalia", Mérida. (3) I. B. "Puente Ajuda", Olivenxa.

(4) I. B. "Antonio Machado", Madrid. (5) Unidad de Programas Educativos.

\section{SUMMARY}

In this paper a display is made of the controversy about the concept of mol and the convenience of introducing it in the school "curricula" at the age in which nowadays is made. The results we have obtained from the research carried out on the difficulties experienced by 15 to 19 year old students in understanding this concept and other related concepts are given.

\section{INTRODUCCIÓN}

En contraste con algunos conceptos físicos, especialmente mecánicos, donde la intuición de los alumnos (Solís 1984), o sus ideas preconcebidas (Leboutet y Barrel 1976) juegan un importante papel, el concepto de mol o la estequiometría de una reacción química son ideas completamente novedosas para nuestros alumnos, que difícilmente puede haberlas intuido previamente en su aprendizaje escolar y que constituyen una fuente de errores conceptuales. Estas cuestiones nos hacen reflexionar sobre dos puntos:

\section{2. ¿EXISTE ALGUNA DIFICULTAD INTRIN- SECA AL CONCEPTO DE MOL?}

La confusión en los químicos del siglo XIX acerca del peso de átomos y moléculas, así como del número de átomos que forman una molécula, fue grande hasta que el químico italiano Cannizaro, en el Primer Congreso Internacional de Química (Karlsruhe, 1860) retomó y defendió la hipótesis de Avogadro como herramienta para la distinción entre peso molecular y peso atómico de elementos gaseosos.
Surgirán después los conceptos de molécula-gramo y átomo-gramo y la conveniencia de determinar en esas porciones de materia el número de moléculas o átomos contenidos. Loschmit sería quien primero lo lograría (1865), obteniendo una valor de $4,1.10^{22}$. Poco después aparecería el concepto de"mol " introducido por Ost." wald (1896), al tiempo que surgían ásperas discusiones en torno a la interpretación y significado del propio concepto, asociado a: unidad individual de masa (es decir, sinónimo de molécula-gramo, fórmula-gramo), porción de sustancia y número de Avogadro.

Más tarde, la I.U.P.A.P. (1958) intentaría unificar criterios proponiendo el mol como: "...la cantidad de sustancia que contiene el mismo número de moléculas (o iones, o átomos) que el existente exactamente en I 6 g del ${ }^{16} \mathrm{O}$ ". Definición que no cerraría la controversia, dado el carácter fuertemente abstracto de "cantidad de sustancia" (Dierks 1981).

La superación de esta dificuitad fue intentada por la I.U.P.A.C. (1967) al proponer el concepto de mol como: "la cantidad de sustancia de un sistema material que contiene tantas entidades elementales como átomos de $\mathrm{C}$ hay en $0,012 \mathrm{~kg}$ de ${ }^{12} \mathrm{C}$. AI usar el concepto 
de mol, las entidades elementales deberán especificarse, pudiendo tratarse de átomos, moléculas, iones, electrones, $u$ otras partículas o agrupaciones especificadas de tales partículas".

A pesar de las concreciones introducidas, tales como "sistema material" o "necesidad de especificar las entidades elementales", continuarán las discusiones en torno a la pretensión de cuantificar la "cantidad de sustancia" en ecuaciones matemáticas (Davies 1973, Kolb 1978), o bien las imprecisiones señaladas de tomar el mol como unidad de masa individual, por ser unidad de valor numérico distinto para distintas sustancias, cuestion esta rechazada por la I.U.P.A.C. (1969 y 1979). Así también, tomar el mol como porción de sustancia, muestra la dificultad de definir la magnitud "cantidad de sustancia" y luego transferirla a cálculos cuantitativos (Cohen 1961), lo que intent6 superarse con la introducción del término "número de moles, $\mathrm{n}$ " (Bradley 1971, Kolb 1978), cuestión criticada por la I.U.P.A.C. que considera "inconsistente llamar ' $n$ ' al número de moles, como lo es llamar ' $m$ ' al número de kilogramos". Por último, el aspecto numérico del con. cepto de mol, presente en la discusión desde los primeros momentos (Lee 1961), si bien debería tenerse en cuenta que el número por sí mismo no es suficiente, ya que en química el mol se refiere a una colección física de objetos (Cohen 1961).

El problema didáctico que se plantea en estas condiciones es arduo (Novick y Menis 1976). El uso del mol en ocasiones como peso o masa, otras cormo volumen, y otras como número, no ayuda a una rápida comprensión por parte de los alumnos, creándoles dificultades que pueden perdurar hasta los primeros niveles universitarios, manifestando con ello que aún no han interio. rizado de forma definitiva el concepto de mol en sus diferentes matices.

Otros hechos apuntados como fuente de errores son la introducción simultánea de "cantidad de sustancia" y de "mol" comparando masas y volúmenes de diferentes porciones de sustancia (Dierks 1981). O también la utilización de un mismo número para expresar magnitudes come masa molecular, mol, masa molar, sin conocer o haber comprendido la relación entre ellas; así como la similitud fonética entre mol, molécula, peso molecular, masa molar, volumen molar..., términos que se les introducen a los alumnos en muy poco tiempo (Novick y Menis 1976).

Gower et al. (1977) señalan cómo los conceptos teóricos son más difíciles de aprender que los empíricos y encuentran que el de "mol" es el teórico de más elevada jerarquía de todos los que subyacen bajo él, y por lo tanto, de los más duros para su aprendizaje.

La introducción del concepto de mol en su aspecto "numérico" es defendida por numerosos autores, ya que facilita los cálculos estequiométricos de las reacciones químicas (Dierks 1981), así como el ajuste de reacciones o el cálculo de molaridad de disoluciones (Johnstone, Morrisson y Sharpe 1971).
Dificultades todas ellas que nos conducen a la segunda cuestión objeto de reflexión.

\section{3. ¿ESTÁN LOS ALUMNOS CAPACITADOS PARA APRENDER EL CONCEPTO DE MOL A LA EDAD QUE SE LES ENSENA?}

Trabajando en la línea abierta por Piaget en torno a la secuencia del desarrollo conceptual, Ingle y Shayer (1971) han indicado que para poder entender en su totalidad el concepto de mol, los alumnos precisan haber alcanzado el nivel conceptual 3B, caracterizado por las capacidades de:

- Comprensión de la proporción múltiple.

- Utilización de símbolos abstractos para resolver problemas.

- Relación entre causa y efectos.

- Emisión y comprobación de hipótesis.

Ahora bien, ¿nuestros alumnos han alcanzado este nivel conceptual cuando se enfrentan al concepto de mol?

Se ha demostrado empíricamente (Mckinson y Reuner 1971) que el $50 \%$ de alumnos de colegios americanos son incapaces de usar las estructuras de razonamiento formal correspondientes al nivel conceptual 3 de Piaget.

La mayoría de los autores coinciden en los porcentajes de alumnos capaces de comprender el concepto de mol. Shayer (1970) señala que sólo el 5-6\% de los niños de 15 años pueden hacerlo. Novick y Menis (1976) indican que la mayoría de sus alumnos no llegan a captar en profundidad este concepto.

\section{OBJETIVOS}

El hecho de ser el mol, en sus diferentes matices, una importante fuente de exror en todo el estudio posterior de la Química, junto con los análisis controvertidos del tema, anteriormente expuestos, y con la escasez de estudios que sobre este asunto hemos encontrado, relacionados con estudiantes de nuestro país, nos han motivado a desarrollar este trabajo de investigación, con la pretensión de conseguir al menos, los siguientes objetivos generales:

1. Conocer cuáles son las ideas que sobre el mol y sus implicaciones poseen los alumnos de BUP, COU y $1^{9}$ de Universidad, al comenzar sus respectivos cursos.

2. Relacionar estas ideas, así como los errores que puedan acompañarlas, con el nivel académico de di- 
chos alumnos, tratando de encontrar su posible relación y evolución curricular.

\section{DISEÑO EXPERIMENTAL}

La poblacion objeto de estudio ha sido la correspondiente a cuatro centros de EE.MM. y dos universitarios, todos ellos de la provincia de Badajoz. Los centros de Enseñanza Media se eligieron de modo que uno fue de ambiente rural, otro semiurbano, otro urbano de la periferia y un último urbano de zona céntrica. En dos de ellos se tomaron datos de alumnos de los regímenes de diurno y nocturno. Uno de los cursos universitarios se tomó de la Facultad de Ciencias (Sección Físicas) y otro de EE.UU. (Formación del Profesorado de EGB). No se controlaron variables de edad, sexo o carácter de repetidor.

La muestra inicial estuvo formada por 650 alumnos, seleccionados mediante procedimiento aleatorio simple, de los que se tomó la muestra final, por el mismo procedimiento, formada por 333 alumnos, distribuidos, según niveles, del modo siguiente:

\begin{tabular}{|c|c|}
\hline $2^{9}$ de BUP & 86 alumnos \\
\hline $3^{9}$ de BUP & $83 \quad$, \\
\hline COU & 96 \\
\hline $1^{\circ}$ Facultad Ciencias & 34 \\
\hline $1^{9}$ Escuela Universitaria & 34 \\
\hline $1^{2}$ Escuela Universitaria & 34 \\
\hline
\end{tabular}

La encuesta utilizada como herramienta de estudio fue pasada en los meses de diciembre-enero. Para su confección se elaboró una batería de 60 cuestiones objetivas, abiertas, de respuesta breve, referentes a los distintos aspectos de interés. Adecuadamente jerarquizados en los diferentes niveles intelectuales requeridos para sus respuestas, se seleccionaron 20 de ellos mediante el procedimiento aleatorio simple, se distribuyeron para su presentación final a los alumnos (ver anexo). En Ia misma se distinguen siete bloques temáticos:

I. Aspectos generales sobre átomos y moléculas (cuestiones $4,6,12$ y 20 ).

II. El mol como unidad de Sistema Internacional (cuestiones 3,7 y 18).

III. Relación número de partículas-masa molar (cuestiones $13,14 a, 17$ y 19 ).

IV. Relación cantidad de sustancia-número de partículas (cuestiones $1,5,9$ y 14c).

V. Relación cantidad de sustancia-masa (cuestiones 10 y 16).

VI. Relacion cantidad de sustancia-volumen molar (cuestiones 4,5 y $14 b$ ).

VII. Estequiometría (cuestiones $1,2,11$ y 15).

\section{RESULTADOS Y DISCUSIÓN}

\section{Bloque I: aspectos generales sobre átomos y molécu- Ias}

Se pretende explorar las ideas de los alumnos en torno a la discontinuidad de la materia, la conservación de partículas en procesos físicos y algunas características de las mismas. En la cuestión $\mathrm{n}^{2} 4$ abordamos dicha conservación, con la $\mathrm{n}^{2} 6$ queremos detectar la diferenciación que hacen entre partículas atómicas y moleculares, con la $\mathrm{n}^{2} 20$ la diferenciación que hacen entre elemento-compuesto y su posible asociación átomomolécula, finalmente con la $n^{2} 12$ analizamos la interpretación que dan a la formula química.

Los resultados se exponen en la tabla I, refiriéndose los de la cuestión no 6 a la molécula $\mathrm{O}_{2}$.

Tabla I

Respuestas correctas deI Bloque I

\begin{tabular}{cccccc} 
Cuest. & 2O BUP & 30 BUP & COU & UN.A & UN.B \\
\hline $4 \mathrm{a}$ & 30,1 & 54,1 & 62,1 & 82,2 & 32,4 \\
$4 \mathrm{~b}$ & 29,7 & 61,1 & 68,7 & 85,4 & 70,6 \\
6 & 40,7 & 65,1 & 75,0 & 97,1 & 88,2 \\
20 & 24,7 & 56,5 & 53,7 & 55,9 & 38,3 \\
12 & 14,9 & 52,5 & 50,0 & 58,8 & 47,1
\end{tabular}

Entre las respuestas erróneas a la cuestión 4a se encuentran mayoritariamente: "la masa del gas aumenta", "disminuye"; y a la ab: "aumenta el número de partículas". La frecuencia de las mismas decrece cuando avanza el currículo (excepto para el Univ. B). En la cuestión $n^{0} 6$, destaca que $S_{8}$ es considerada como átomo por gran número de alumnos (alrededor del $20 \%$ en todos los niveles salvo Univ. A. con el 33\%). En la cuestión $n^{2} 20$ las especies $\mathrm{O}_{2}, \mathrm{H}_{2}$ y $\mathrm{S}_{8}$ son consideradas como compuestos ( $20 \%$ de alumnos). En la cuestión $n^{\circ}$ 12 se aprecia una gran dispersion de respuestas, observándose un escasísimo grado de comprensión del lenguaje utilizado en la simbología química en ahumnos de $2^{0}$ de BUP. En otros cursos se obtienen mejores resultados, pero la evolución del aprendizaje significativo conseguido no es muy satisfactoria.

\section{Bloque II: el mol como unidad del sistema interna- cional}

Dado que el concepto "cantidad de sustancia" aparece asociado al de masa o volumen, y que en muchos libros de texto se presenta el mol como otra unidad más de masa ( $y$ no como una unidad de la magnitud cantidad de sustancia), no parece extraño que esta asociación se manifieste también en los alumnos. En la cuestión n ${ }^{2} 3$ se aborda directamente la idea del concepto de mol. En la cuestión $n^{\circ} 18$ se plantea la aplicación de la misma a un caso concreto, y en la cuestión $n^{\circ} 7$ al alumno se le 
pide establecer los rasgos comunes de un mol de sustancias distintas. Los resultados se recogen en la tabla II.

Tabla II

Respuestas correctas a las cuestiones del Bloque II

\begin{tabular}{|c|c|c|c|c|c|}
\hline Cuest. & 20 BUP & 3 으 BUP & $\operatorname{cou}$ & UN.A & UN.B \\
\hline 3 & -- & 27,7 & 18,8 & 32,4 & 14,7 \\
\hline 18 & -- & 16,0 & 19,1 & 38,2 & 8,8 \\
\hline 7 & 3,5 & 23,2 & 58,3 & 41,2 & 23,5 \\
\hline
\end{tabular}

En la cuestion no 3 aparece una elevada dispersión, siendo frecuente encontrar entre las respuestas erróneas, que discrepan de la definición de la I.U.P.A.C., la iđentificación mol-masa molar: "peso molecular expresado en gramos", situación que se intensifica con el currículo, o bien se asocia a volumen molar de gases en condiciones normales. En la cuestión no 18 el porcentaje de respuestas erróneas es muy alto. Como respuestas erróneas aparecen: " 1 molécula de agua", "22,41 de agua", "cantidad de agua igual a un peso molecular", "2 atomos de $\mathrm{H}$ y 1 de $\mathrm{O}$ ", etc. Si comparamos estos valores con los obtenidos en la cuestión anterior, podemos corroborar el hecho de la superficialidad del aprendizaje de conceptos por parte de algunos alumnos: son capaces de memorizar una definición, pero tienen serios problemas a la hora de aplicarla a casos concretos.

En la cuestión $n^{9} 7$ aparecen también como respuestas, además de la correcta (mismo número de partículas): "mismo número de moléculas", "mismo número de átomos", "nada", "ocupan el mismo volumen". Como se ve, los alumnos apuntan hacia el número de entidades como rasgo común del mol, si bien no precisan el tipo de partícula que caracteriza a cada sustancia. Quizás las especificaciones de la entidad respectiva al hablarles del mol podría clarificar esto a los alumnos.

\section{Bloque III: relación número de partículas-masa molar}

Pretendemos profundizar en el conocimiento del "carácter ambivalente" con el que los alumnos utilizan el mol. En la cuestión no 13 relacionamos el número de átomos contenidos en una determinada masa de cierta sustancia con su masa atómica. En la n ${ }^{\circ} 19$, abordamos directamente el concepto de masa molar. La n ${ }^{8} 14 \mathrm{a}$ es una aplicación de la anterior a un caso concreto. En la $\mathrm{n}^{\mathrm{Q}} 17$ se propone la operación inversa. Los resultados se recogen en la tabla III.

En la cuestión i3 se observa un progresivo afianzamiento del concepto de masa atómica como propiedad del átomo. Los alumnos de $2^{\circ}$ y $3^{2}$ de BUP responden directamente, mientras que los de COU y los universitarios, además lo hacen operativamente. En la cuestión no 19 resaltan los bajos resultados, así como cierta confusión entre masa molar y masa molecular. Entre las respuestas erróneas a la $14 a$ se encuentra " 10 átomos". Dada la sencillez de la operatividad requerida, los pobres resultados obtenidos en este bloque apuntan hacia una complejidad de estos conceptos, inicialmente insospechada. Las entidades químicas átomos o moléculas, quizá por su imposibilidad de percepción directa, son suficientemente enigmáticos para los alumnos. Creemos que el uso de las masas absolutas de dichas entidades, en lugar de las relativas, puede colaborar muy positivamente a concretarlas. Proponemos que la masa del átomo o molécula correspondiente sea acompañada del símbolo internacional de la unidad de masa atómica (n), y no de su abreviatura (u,a.m.).

Tabla III

Respuestas correctas a las cuestiones del Bloque III

\begin{tabular}{lccccc} 
Cuest. & 20 BUP & 3O BUP & COU & UN.A & UN.B \\
\hline 13 & 9,2 & 41,0 & 68,1 & 94,1 & 50,0 \\
19 & 18,4 & 44,6 & 57,3 & 76,5 & 44,1 \\
$14 a$ & 1,1 & 13,7 & 63,0 & 73,5 & 38,2 \\
17 & $-\infty$ & 19,3 & 48,9 & 79,4 & 23,6
\end{tabular}

\section{Bloque IV: relación cantidad de sustancia-n ${ }^{2}$ de partículas}

Para saber el grado de conocimiento de los alumnos del número de Avogadro y su asociación con el $n^{9}$ de entidades químicas contenidas en cierta cantidad de sustancia, se le plantea directamente el problema en la cuestión $n^{\circ} 9$, a través de cierta perífrasis en la $n^{\circ} 14 \mathrm{c}$ y el cálculo inverso en la $n^{\circ} 5$. Los resultados correctos se recogen en la tabla IV.

Los alumnos de $2^{2}$ de BUP manifiestan un claro desconocimiento del tema, observándose un salto cuantitativo importante entre éste y $3^{\circ}$ de BUP. Las respuestas correctas a la cuestión $14 \mathrm{c}$ son sensiblemente inferiores al resto, quizás por la mayor complejidad del enunciado. Algunas respuestas incorrectas a la cuestión 9 apuntan obstinađamente al $\mathrm{n}^{2} 12$ : "12 átomos", "12 NA", "12 gramos", "12". Entre las dadas a la $14 \mathrm{c}$ destaca: "0,5 átomos" ( $10 \% 3^{\circ}$ de BUP y $14,7 \%$ Univ. B).

Tabla IV

Respuestas correctas a las cuestiones del Bloque IV

\begin{tabular}{cccccc} 
Cuest. & 20 BUP & 30 BUP & COU & UN.A & UN.B \\
\hline 9 & -- & 43,4 & 70,8 & 85,3 & 47,1 \\
$14 \mathrm{c}$ & -- & 11,2 & 61,0 & 76,5 & 23,5 \\
5 & 2,3 & 42,2 & 86,3 & 94,2 & 79,4
\end{tabular}




\section{Bloque V: relación cantidad de sustancia-masa}

Mecanismos aparentemente triviales como la identificación masa atómica (o molecular) con la masa de un solo átomo (o molécula), la asociación masa de una molécula con la masa de un mol de moléculas, o el establecimiento de proporciones adecuadas entre la masa de un mol y otra masa de la misma sustancia, son las que se exploran en este bloque cuyos resultados se recogen en la tabla V.

Tabla V

Respuestas correctas a las cuestiones del Bloque V.

\begin{tabular}{llllll} 
Cuest. & 2o BUP & 30 BUP & COU & UN.A & UN.B \\
\hline 16 & 5,8 & 44,6 & 87,3 & 91,2 & 52,9 \\
10 & 2,3 & 56,6 & 82,1 & 82,4 & 58,9
\end{tabular}

En la cuestión 16, un importante porcentaje de alumnos llega a la respuesta de forma directa o mediante una sencilla división, donde no hacen intervenir unidades, y a veces expresiones erróneas o unidades no coherentes: " $42,5 \mathrm{~g} / 17$ uma", "masa/peso molecular". En Ia n" 10 aparecen respuestas como: "mayor para el $\mathrm{CO}_{2}$, menor para el $\mathrm{N}_{2}$ " o "todas iguales". Análogamente al caso anterior, los cálculos son generalmente rutinarios, sin que parezcan denotar otras consideraciones conceptuales de los alumnos.

\section{Bloque VI: relación cantidad de sustancia-volumen molar}

El concepto de volumen molar (volumen de un mol) suele explicarse asociado a gases en condiciones normales, lo que suele ser fuente de errores y respuestas rutinarias, como encontramos en las respuestas a este bloque, cuyos resultados se recogen en la tabla VI.

Tabla VI

Respuestas correctas a las cuestiones del Bloque VI.

\begin{tabular}{cccccc} 
Cuest. & 20 BUP & 30 BUP & COU & UN.A & UN.B \\
\hline 8 & 22,9 & 12,0 & 26,6 & 61,8 & 55,9 \\
$14 \mathrm{~b}$ & -- & 8,7 & 54,3 & 76,5 & 29,4 \\
\hline
\end{tabular}

Respuestas frecuentes a la cuestion $n$ ㅇ 8 son: "volumen de un mol en c.n." o "volumen de un mol de gas" (especificando, o no, las condiciones). En $2^{\circ}$ de BUP quizás puedan existir aciertos por "deducción semántica"; en los cursos inferiores hay cierta tendencia a confundir volumen molar con volumen molecular; en los universitarios, la confusión es con la concentración molar. En la cuestión $14 b$ se aprecia un importante desconocimiento, habiéndose encontrado respuestas como: "I átomo" ( $20 \% 3^{\circ}$ de BUP, $12 \%$ Univ. B).

\section{Bloque VII: estequiometría}

Abordamos aspectos fundamentales de la estequiometría tales como la percepción de la información subyacente en una ecuación química, cuestión $\mathrm{n}^{\circ} 2$, conservación de la masa, cuestión $n^{\circ} 15$, sencillos cálculos estequiométricos, en la $n^{2} 1$, y ley de Proust cuando los reactivos no se encuentran en la proporción de combinación. Los resultados se muestran en la tabla VII.

Tabla VII

Respuestas correctas a las cuestiones del Bloque VH

\begin{tabular}{rrrrrr} 
Cuest. & 20 BUP & 30 BUP & COU & UN.A & UN.B \\
\hline 2 & 3,4 & 53,1 & 75,0 & 55,9 & 38,2 \\
15 & 2,4 & 40,1 & 57,9 & 67,7 & 55,8 \\
1 & -- & 39,8 & 70,9 & 94,1 & 41,2 \\
11 & 9,3 & 36,2 & 45,7 & 76,5 & 11,8
\end{tabular}

En las respuestas a la cuestión $\mathrm{n}^{\circ} 2$ se aprecia una mejora progresiva que "rompen" los alumnos universitarios con: "es una reacción química". En general hacen pocas alusiones al estado físico de las sustancias, clasifican el proceso de "disociación", "combustión", "evaporación de oxígeno", "cambio de estado", etc. y particularmente en cursos inferiores consideran con frecuencia al coeficiente estequiométrico parte de la entidad química. En la cuestión $n^{\circ} 15$, las respuestas presentan una elevada dispersión y suelen ser incompletas o imprecisas: "para que quede equilibrada", "para que a ambos lados haya el mismo número de moléculas", "porque si no, el compuesto no se forma". En la cuestión $n^{\circ} 1$, altos porcentajes, en particular Univ. B (38\%), responden: "I molécula". En la cuestión $\mathrm{n}^{\circ} 11$, es muy frecuente la respuesta: "19 gramos" (52\% BUP, $45 \% 3^{\circ}$ de BUP, $26 \%$ COU, $6 \%$ Univ. A, $68 \%$ Univ. B); parece prevalecer la Ley de Lavoisier sobre la de Proust, siendo muy pocos los alumnos que indican el exceso de hidrógeno; otros dan masas superiores a la de reactivos, o bien señalan la imposibilidad de la reacción (algunos alumnos de COU y Univ. B).

\section{CONCLUSIONES}

$2^{\circ}$ BUP: Los resultados muestran un escasísimo grado de conocimiento (generalmente nulos o inferior al $5 \%$ de respuestas correctas), salvando las obtenidas en eI Bloque I, sin duda el más elementaI, y en los Bloques III y IV las respuestas a masa y volumen molar (adjudicables más a razones lingüísticas que de conocimiento). Dado que estos alumnos ya tomaron contacto con 
el comportamiento químico de la materia ( $8^{\circ}$ de EGB) el salto hasta $2^{\circ}$ de BUP parece producir un serio quebranto en sus conocimientos, por ello la enseñanza de la Química de $2^{\circ}$ de BUP debería tomar en consideración este hecho, e incluso preguntarse si dada la difícultad que parece observarse en la progresiva adquisición e interiorización de muchos conceptos, no convendría introducirlos ya en el BUP, poniendo mayor énfasis en los aspectos cualitativos de los sistemas químicos en $8^{\circ}$ de EGB.

$3^{\circ}$ BUP: Los porcentajes de respuestas correctas mejoran sensiblemente respecto a tos anteriores, aunque generalmente apenas sobrepasan el 50\% (salvo en el Bloque I). Esto apunta hacia la mejora en el nivel de conocimiento y comprension que para estos alumnos ha supuesto un paso por $2^{\circ}$ de BUP. A pesar de ello, no debe olvidarse la elevada dispersión que se encuentra en sus respuestas, algunas de las cuales recaen en importantes errores conceptuales.

COU: Sigue acentuándose la mejora de resultados, habiéndose reducido considerablemente las respuestas en blanco y la dispersión del resto. Sin embargo, los resultados no pueden considerarse excesivamente buenos si se tiene en cuenta que en el momento de pasar la encuesta estos alumnos ya habían tratado estos temas. A veces se aprecia no sólo la persistencia, sino incluso la acentuación de algún error conceptual como la identificación de mol de una sustancia con su masa molar.

Grupo universitario A: Corresponde este grupo al primer curso de la Facultad de Ciencias Físicas, y como era de esperar, es el que mejores resultados presenta. La dispersión en sus respuestas es generalmente baja y sus respuestas en blanco escasas. Se detectan algunas dificultades en estos alumnos en la resolución de cuestiones con enunciado largo y mezcla de conceptos varios, así como en la comprensión y uso de razonamiento de proporcionalidad inversa. Algunos errores se muestran persistentes, entre ellos Ia no conservación de la masa y partículas en cambios físicos y la fuerte asociación mol-masa molar. Presentan una mayor facilidad para la resolución correcta de cuestiones operativas que para las conceptuales, lo que deja abierta la duda sobre que estos cálculos tengan un gran componente rutinario.

Grupo universitario B: Los componentes de este grupo son alumnos de Primer Curso de la Especialidad de Ciencias de la E.U.F.P. de EGB. Las respuestas correctas que presentan son considerablemente bajas, para su nivel, en muchos casos ante preguntas sencillas. Los resultados, inesperadamente, aparecen, casi siempre, por debajo de los de COU, mostrando un notable parecido con los de alumnos de $3^{\circ}$ de BUP.

\section{Nota}

Esta exposición es un resumen de la memoria de investigación "Percepción de conceptos básicos en Química y su evolucion en alumnos de 15-19 años", realizada por el Grupo Alkali bajo la dirección del Dr. J. P. García Martín, con la subvención del CEP-Badajoz, al que agradecemos la ayuda prestada. 


\section{ANEXO: Cuestiones}

1.- En la reacción $\mathrm{CaCO}_{3}(\mathrm{~s}) \rightarrow \mathrm{CaO}(\mathrm{s})+\mathrm{CO}_{2}$ (g), ¿cuántas moléculas de $\mathrm{CO}_{2}$ se obtienen a partir de $1 \mathrm{~mol}^{2}$ de $\mathrm{CaCO}_{3}$ ?

2.- ¿Qué expresa para ti la siguiente ecuación química?: $2 \mathrm{KClO}_{3}(\mathrm{~s}) \rightarrow 2 \mathrm{KCl}(\mathrm{s})+3 \mathrm{O}_{2}(\mathrm{~g})$

3.- ¿Qué entiendes por mol?

4.- $100 \mathrm{I}$ de un gas en condiciones normales tienen una masa de $50 \mathrm{~g}$. Se calienta el gas y su volumen aumenta hasta $200 \mathrm{l}$. a.- ¿Que le ocurrirá a la masa del gas?

b.- ¿Qué le ocurrirá al número de partículas del mismo?

5.- Cuántos moles de sodio son $12,046.12^{23}$ átomos de dicho elemento?

6.- Según tu concepción de átomos y moléculas, clasifica como tales cada una de las siguientes sustancias: $\mathrm{H}, \mathrm{H}_{2} \mathrm{O}$, $\mathrm{HCl}, \mathrm{O}_{2}, \mathrm{H}_{2}, \mathrm{~S}_{3}, \mathrm{Fe}, \mathrm{H}_{2} \mathrm{SO}_{4}$.

7.- ¿Tienen algo en común $1 \mathrm{~mol}$ de agua y 1 möl de hierro?

8.- ¿Qué entiendes por volumen molar?

9.- ¿Qué número de partículas contiene $1 \mathrm{~mol}$ de carbono?

10.- Las masas moleculares de las siguientes sustancias: $\mathrm{HCl}, \mathrm{N}_{2}$ y $\mathrm{CO}_{2}$, son respectivamente $36.5,28$ y 44 u. $\mathrm{Si}$ consideramos $100 \mathrm{~g}$ de cada una đe ellas, señala cuál contiene mayor y cuál menor número de moles.

$11,-8 \mathrm{~g}$ de oxígeno reaccionan con $1 \mathrm{~g}$ de hidrógeno para dar $9 \mathrm{~g}$ de agua. Si reaccionan $16 \mathrm{~g}$ de oxígeno con $3 \mathrm{~g}$ de hidrógeno, ¿cuántos gramos de agua se formarán?
12.- ¿Qué significa para ti que el ácido sulfúrico tenga đe formula $\mathrm{H}_{2} \mathrm{SO}_{4}$ ?

13.- Al comparar el número de átomos existentes en I $\mathrm{g}$ de carbono y los existentes en $1 \mathrm{~g}$ de sodio, ¿cómo piensas que será el resultado (igual, mayor o menor)?

(Masa atómica del carbono: $12 \mathrm{u}$, del sodio: $23 \mathrm{u}$ ).

14.-- El helio es un gas monoatómico. Su masa molar es $4 \mathrm{~g} /$ mol iCuántos átomos de helio hay en las siguientes cantidades del mismo?

a. $-40 \mathrm{~g}$

b. $-22,4$ I en condiciones normales

c. $-0,05$ moles

15.- La reacción química $\mathrm{N}_{3}+\mathrm{H}_{2} \rightleftharpoons \mathrm{NH}_{3}$ se iguala dando la ecuación: $\mathrm{N}_{2}+3 \mathrm{H}_{2} \rightleftharpoons 2 \mathrm{NH}_{3}$. ¿Por que se han de igualar las reacciones químicas?

16.- La masa molecular del amonfaco es 17 u. ¿Cuántos moles de estas sustancia hay ent $42,5 \mathrm{~g}$ de la misma?

17.-La masa molar de la sustancia X es de $300 \mathrm{~g} / \mathrm{mol}_{\text { ¿ Cuál }}$ es Ia masa de una sola partícula (o unidad fundamental) de X?

18.- ¿Qué es para ti un mol de agua?

19.- ¿Qué entiendes por masa molar?

20.- Clasifica las sustancias de la cuestión número 6 como elementos químicos o compuestos.

\section{REFERENCIAS BIBLIOGRÁFICAS}

BRADLEY, W. G., 1971. A definition of the mole suitable for physics, The Physic Teacher, 9, pp. 358-387.

COHEN, I., 1961. Moles and equivalents: quantities of matter, Journal of Chemical Education, 38, pp. 555-556.

DAVIES, P. O., 1973. Avogadro's number and Avogadro's constant, Physics Education, 5, pp. 275-277.

DIERKS, W., 1981. Teaching the mole, European Journal of Science Education, 3, p. 145.
GOWER, D. M., DANIEL'S, D. I., y LLOYD, G., 1977. Hierarchies among the concepts wich underlie the mole, School Science Review, 59, pp. 285-299.

INGLE, R. B. y SHAYER, M., 1971. Nuffiel O-level chemistry, Education in Chemistry, 8, pp. 182-183.

IOHNSTONE, A. A., MORRISON, J. I, y SHARPE, D. W. A., 1971. Topic difficulties in chemistry, Education in Chemistry, 8, pp. 212-213. 
KOLB, D., 1978. The mole, Journal of Chemical Education, 55, pp. 728,732 .

LEBOUTET, L. y BARREL, 1976. Concepts of mechanics among young people, Physics Education, 11, pp. 462-465.

LEE, S., 1961. A redefinition of mole, Journal of Chemical Education, 38, pp. 549-551.

MACKINSON, J. W, y RENNER, W., 1971. Are colleges concerned with intellectual Development?, American
Journal of Physics, 39, p. 1.047.

NOVICK, S. y MENIS, J., 1976. A study of students perceptions of the mole concept, Journal of Chemical Education, $53, \mathrm{pp} .720-722$.

SHAYER, M., 1970. How to assess science courses?, Education in Chemistry, 10, p. 182.

SOLIS, R, 1984. Ideas intuitivas y aprendizaje de las ciencias, Enseñanza de las Ciencias, 2, pp. 83-89. 


\title{
UNA ANALOGÍA TÉRMICA PARA LA ENSENANZA DE LA CORRIENTE CONTINUA EN ELECTRICIDAD: DESCRIPCIÓN Y EVALUACIÓN
}

\author{
DUPIN, J. J. y JOSHUA, S. \\ Universidad de Provence. \\ Facultad de Ciencias de Marsella.
}

Traducción de C. Furió Mas

\section{SUMMARY}

This paper reports the application of thermical analogy to the teaching of electrical current to secondary students. At the same time the students' cognitive processes on this subject are commented.

\section{INTRODUCCIÓN}

\subsection{Las anajogías en la enseñanza}

El niño no construye su saber simplemente por acurnulación de capas sucesivas de conocimientos, sino a través de un proceso personal y continuo de reconstrucción de lo nuevo a partir de lo viejo. Avances importantes se realizan cuando aparecen posibilidades de reestructuración, poniendo en relación dominios de conocimiento antes disjuntos. El razonamiento analógico es uno de los medios que permiten establecer estas relaciones. Precisemos primero lo que entendemos por "analogía", sirviéndonos para ello de la definición de Kircher (1977). Consideremos un objeto O y el modelo $\mathrm{M}$ que lo describe. Puede existir otro par (objeto $O^{\prime}$ ) (modelo $\mathbf{M}^{\prime}$ ) tal que existen entre los modelos $\mathrm{M}^{\text {y }} \mathbf{M}^{\prime}$ unas relaciones de estructura isomorfas. Los modelos M y $\mathbf{M}^{\prime}$ se đice, entonces, que son análogos.

Cuando se presenta una de estas situaciones en cualquier dominio de la enseñanza, la utilización de estas analogías puede proporcionar una ayuda apreciable al profesor.

Para estudiar una nueva pareja O.M el profesor puede recurrir a otro par analógico $O^{\prime} . M^{\prime}$ ' si le es más familiar a sus alumnos. Puede esperar así que se realice la transferencia de las relaciones establecidas entre $O^{\prime}$ $\mathrm{M}^{\prime}$ hacia el par O-M. Esto ya ha sido estudiado por muchos autores que han puesto de relieve tanto los logros como las dificultades encontradas: Transferencia de un par a otro (Tenney y Gentner 1984), dificultades semejantes en la comprehensión de conceptos en los dos modelos (Johnstone y Mughol 1976), analogías negativas (Hesse 1966), sustitución del objeto real de enseñanza (Kircher 1984).

\subsection{La analogía "modelizante"}

Presentamos aquí la utilización, en la enseñanza de la corriente continua en electricidad, de su analogía con la conducción térmica.

Esta analogia, muy clásica, ha sido según nuestros conocimientos mucho menos empleada que la analogía hidráulica. Nos ha parecido interesante estudiarla des. de el punto de vista didáctico para analizar su pertinen cia en situación de clase. Teniendo en cuenta las dificultades mencionadas anteriormente, debemos tomar ciertas precauciones: 